\title{
Hardy-type inequalities associated with the Weinstein operator
}

Hatem Mejjaoli*

Dedicated to my Father Youssef Mejjaoli

"Correspondence:

hatem.mejjaoli@yahoo.fr Department of Mathematics,

College of Sciences, Taibah University, P.O. Box 30002

Al Madinah Al Munawarah, Saudi Arabia

\section{Springer}

\begin{abstract}
In this article, we define and we study the Hardy spaces associated with the Weinstein operator. We establish refined Sobolev inequalities between the homogeneous Weinstein-Besov spaces and many spaces, as the homogeneous Weinstein-Riesz spaces and the generalized Lorentz spaces. Next we prove Hardy-type inequalities in the homogeneous Weinstein-Besov space, homogeneous Weinstein-Riesz space, and Weinstein-Hardy space.
\end{abstract}

MSC: Primary 35L05; secondary 22E30

Keywords: Weinstein operators; Weinstein-Hardy spaces; Hardy-type inequalities; inhomogeneous Weinstein-Besov spaces; generalized Lorentz spaces

\section{Introduction}

The theory of classical real Hardy spaces in $\mathbb{R}^{d}$ originates from the study of holomorphic functions of one variable in the upper half-plane. For characterizations and properties of the classical Hardy spaces we refer the reader to the original work [1-3]. Hardy spaces on spaces of homogeneous type (see, e.g., $[4,5]$ ) are extensions of the classical real Hardy spaces on $\mathbb{R}^{d}$. More information is given in [6] and the references therein. We note that the subject of the generalized Hardy spaces associated with some operators was studied by many authors (see [7-10], among others).

A well-known result of Hardy states that if $f \in H^{p}\left(\mathbb{R}^{d}\right), 0<p \leq 1$, then the inequality of the Euclidean Fourier transform is

$$
\int_{\mathbb{R}^{d}} \frac{|\mathcal{F}(f)(x)|^{p}}{\|x\|^{d(2-p)}} d x \leq C\|f\|_{H^{p}\left(\mathbb{R}^{d}\right)}^{p}
$$

Analogs of Hardy's inequality in the context of eigenfunction expansions have been considered by several authors. In [11] Colzani-Travaglini established a Hardy inequality for eigenfunction expansions associated to the Laplace-Beltrami operator on compact Riemannian manifolds. They have also treated compact symmetric spaces. Kanjin [8] proved Hardy's inequality for the one-dimensional Hermite and Laguerre expansions of the functions $f$ from $H^{1}(\mathbb{R})$. Later, Radha and Thangavelu [12] treated Hardy's inequalities and proved them for higher-dimensional Hermite and special Hermite expansions of func-

(c) 2015 Mejjaoli. This article is distributed under the terms of the Creative Commons Attribution 4.0 International License (http://creativecommons.org/licenses/by/4.0/), which permits unrestricted use, distribution, and reproduction in any medium, provided you give appropriate credit to the original author(s) and the source, provide a link to the Creative Commons license, and indicate if changes were made. 
tions in Hardy spaces. Inequalities for multiple Laguerre expansions are also deduced in the case of Laguerre expansions.

In this paper, we consider the Weinstein operator defined on $\mathbb{R}^{d} \times(0, \infty)$ by

$$
\begin{aligned}
\triangle_{\beta} & :=\sum_{i=1}^{d+1} \frac{\partial^{2}}{\partial x_{i}^{2}}+\frac{2 \beta+1}{x_{d+1}} \frac{\partial}{\partial x_{d+1}}, \quad \beta>-\frac{1}{2} \\
& =\triangle_{d}+\mathcal{L}_{\beta},
\end{aligned}
$$

where $\Delta_{d}$ is the Laplacian for the $d$ first variables and $\mathcal{L}_{\beta}$ the Bessel operator for the last variable, given by

$$
\mathcal{L}_{\beta}=\frac{\partial^{2}}{\partial x_{d+1}^{2}}+\frac{2 \beta+1}{x_{d+1}} \frac{\partial}{\partial x_{d+1}}, \quad \beta>-\frac{1}{2}
$$

For $d>2$, the operator $\Delta_{\beta}$ is the Laplace-Beltrami operator on the Riemanian space $\mathbb{R}^{d} \times$ $(0, \infty)$ equipped with the metric

$$
d s^{2}=x_{d+1}^{2(2 \beta+1) /(d-1)} \sum_{i=1}^{d+1} d x_{i}^{2}
$$

(cf. [13]). The Weinstein operator $\triangle_{\beta}$ has several applications in pure and applied mathematics especially in fluid mechanics (cf. [14]).

The harmonic analysis associated with the Weinstein operator is studied by Ben Nahia and Ben Salem $(c f .[13,15])$. In particular the authors have introduced and studied the generalized Fourier transform associated with the Weinstein operator. This transform is called the Weinstein transform.

Very recently, many authors have investigated the behavior of the Weinstein transform with respect to several problems already studied for the Fourier transform; for instance, Paley-Wiener theorems [16], the Bockner-Hecke theorem [17], uncertainty [18], the Gabor transform [19], Heisenberg-type inequalities [20], and so on.

In the present paper, we intend to continue our study of generalized spaces of type Sobolev associated with the Weinstein operator started in [21]. Indeed, in [21] the author provided a general theory for the Littlewood-Paley case associated with the Weinstein operators. Furthermore, some function spaces associated with Weinstein operators: homogeneous generalized Bessel potential spaces, homogeneous Besov spaces associated with Weinstein operator are studied. We obtain their basic properties and apply them to an estimate of the solutions of the Weinstein heat equation.

The first subject of this paper is to address and analyze in this new context the atomic Hardy spaces in the spirit of the classical scheme. The second subject is to establish Hardytype inequalities between the homogeneous Weinstein-Besov spaces and many spaces, as the homogeneous Weinstein-Riesz spaces and the generalized Lorentz spaces.

The contents of the paper is as follows. In Section 2 we recall some basic results as regards the harmonic analysis associated with the Weinstein operator. In Section 3, we give an appropriate definition of atoms and investigate the atom characterization of Hardy spaces associated with the Weinstein operator. Next we establish many estimates for the Weinstein transform of the functions in the generalized Hardy spaces. In Section 4 we 
establish a Hardy type inequality in the homogeneous Weinstein-Riesz spaces. Next, we introduce the generalized Lorentz spaces associated with the Weinstein operator. We prove refined Sobolev inequalities between the homogeneous Weinstein-Besov spaces and the generalized Lorentz space. Finally, Hardy-type inequalities in the homogeneous Weinstein-Besov spaces and the generalized Hardy spaces are proved.

Throughout this paper, the letter $C$ indicates a positive constant, not necessarily the same in each occurrence.

\section{Preliminaries}

In order to confirm the basic and standard notations we briefly overview the Weinstein operator and related harmonic analysis. The main references are [13, 15]. In this section we collect some notations and results on the Weinstein kernel, the Weinstein transform, and the Weinstein convolution.

In the following we denote by

$$
\begin{aligned}
& \mathbb{R}_{+}^{d+1}=\mathbb{R}^{d} \times[0, \infty), \\
& x=\left(x_{1}, \ldots, x_{d}, x_{d+1}\right)=\left(x^{\prime}, x_{d+1}\right) \in \mathbb{R}_{+}^{d+1} .
\end{aligned}
$$

$C_{*}\left(\mathbb{R}^{d+1}\right)$ the space of continuous functions on $\mathbb{R}^{d+1}$, even with respect to the last variable.

$C_{*}^{p}\left(\mathbb{R}^{d+1}\right)$ the space of functions of class $C^{p}$ on $\mathbb{R}^{d+1}$, even with respect to the last variable.

$\mathcal{E}_{*}\left(\mathbb{R}^{d+1}\right)$ the space of $C^{\infty}$-functions on $\mathbb{R}^{d+1}$, even with respect to the last variable. $\mathcal{S}_{*}\left(\mathbb{R}^{d+1}\right)$ the Schwartz space of rapidly decreasing functions on $\mathbb{R}^{d+1}$, even with respect to the last variable.

$D_{*}\left(\mathbb{R}^{d+1}\right)$ the space of $C^{\infty}$-functions on $\mathbb{R}^{d+1}$ which are of compact support, even with respect to the last variable.

$\mathcal{S}_{*}^{\prime}\left(\mathbb{R}^{d+1}\right)$ the space of temperate distributions on $\mathbb{R}^{d+1}$, even with respect to the last variable. It is the topological dual of $\mathcal{S}_{*}\left(\mathbb{R}^{d+1}\right)$.

We consider the Weinstein operator $\triangle_{\beta}$ defined by $\forall x=\left(x^{\prime}, x_{d+1}\right) \in \mathbb{R}^{d} \times(0, \infty)$,

$$
\triangle_{\beta} f(x)=\triangle_{x^{\prime}} f\left(x^{\prime}, x_{d+1}\right)+\mathcal{L}_{\beta, x_{d+1}} f\left(x^{\prime}, x_{d+1}\right), \quad f \in C_{*}^{2}\left(\mathbb{R}^{d+1}\right),
$$

where $\triangle_{x^{\prime}}$ is the Laplace operator on $\mathbb{R}^{d}$, and $\mathcal{L}_{\beta, x_{d+1}}$ the Bessel operator on $(0, \infty)$ given by

$$
\mathcal{L}_{\beta, x_{d+1}}:=\frac{d^{2}}{d x_{d+1}^{2}}+\frac{2 \beta+1}{x_{d+1}} \frac{d}{d x_{d+1}}, \quad \beta>-\frac{1}{2} .
$$

The Weinstein kernel $\Lambda$ is given by

$$
\Lambda(x, z):=e^{i\left\langle x^{\prime}, z^{\prime}\right\rangle} j_{\beta}\left(x_{d+1} z_{d+1}\right), \quad \text { for all }(x, z) \in \mathbb{R}^{d+1} \times \mathbb{C}^{d+1},
$$

where $j_{\beta}\left(x_{d+1} z_{d+1}\right)$ is the normalized Bessel function. The Weinstein kernel satisfies the following properties: 
(i) For all $z, t \in \mathbb{C}^{d+1}$, we have

$$
\Lambda(z, t)=\Lambda(t, z) ; \quad \Lambda(z, 0)=1 \quad \text { and } \quad \Lambda(\lambda z, t)=\Lambda(z, \lambda t), \quad \text { for all } \lambda \in \mathbb{C}
$$

(ii) For all $v \in \mathbb{N}^{d+1}, x \in \mathbb{R}^{d+1}$, and $z \in \mathbb{C}^{d+1}$, we have

$$
\left|D_{z}^{v} \Lambda(x, z)\right| \leq\|x\|^{|v|} \exp (\|x\|\|\operatorname{Im} z\|)
$$

where $D_{z}^{v}=\frac{\partial^{v}}{\partial z_{1}^{v_{1}} \cdots \partial z_{d+1}^{v} d+1}$ and $|v|=v_{1}+\cdots+v_{d+1}$. In particular

$$
|\Lambda(x, y)| \leq 1, \quad \text { for all } x, y \in \mathbb{R}^{d+1}
$$

The Weinstein intertwining operator is the operator $\mathcal{R}_{\beta}$ defined on $C_{*}\left(\mathbb{R}^{d+1}\right)$ by

$$
\mathcal{R}_{\beta} f\left(x^{\prime}, x_{d+1}\right)= \begin{cases}\frac{\Gamma(\beta+1)}{\sqrt{\pi} \Gamma\left(\beta+\frac{1}{2}\right)} x_{d+1}^{-2 \beta} \int_{-x_{d+1}}^{x_{d+1}}\left(x_{d+1}^{2}-t^{2}\right)^{\beta-\frac{1}{2}} f\left(x^{\prime}, t\right) d t, & x_{d+1}>0 \\ f\left(x^{\prime}, 0\right), & x_{d+1}=0\end{cases}
$$

We note that the Weinstein kernel has the following representation

$$
\forall(x, z) \in \mathbb{R}^{d+1} \times \mathbb{C}^{d+1}, \quad \Lambda(x, z)=\mathcal{R}_{\beta}\left(e^{\langle i x, \cdot\rangle}\right)(z) .
$$

We denote by $L_{\beta}^{p}\left(\mathbb{R}_{+}^{d+1}\right)$ the space of measurable functions on $\mathbb{R}_{+}^{d+1}$ such that

$$
\begin{aligned}
& \|f\|_{L_{\beta}^{p}\left(\mathbb{R}_{+}^{d+1}\right)}=\left(\int_{\mathbb{R}_{+}^{d+1}}|f(x)|^{p} d \mu_{\beta}(x) d x\right)^{\frac{1}{p}}<\infty, \quad \text { if } 1 \leq p<\infty, \\
& \|f\|_{L_{\beta}^{\infty}\left(\mathbb{R}_{+}^{d+1}\right)}=\operatorname{ess} \sup _{x \in \mathbb{R}_{+}^{d+1}}|f(x)|<\infty,
\end{aligned}
$$

where $d \mu_{\beta}$ is the measure on $\mathbb{R}_{+}^{d+1}$ given by

$$
d \mu_{\beta}\left(x^{\prime}, x_{d+1}\right):=x_{d+1}^{2 \beta+1} d x^{\prime} d x_{d+1} \text {. }
$$

The Weinstein transform is given for $f$ in $L_{\beta}^{1}\left(\mathbb{R}_{+}^{d+1}\right)$ by

$$
\mathcal{F}_{W}(f)(y)=\int_{\mathbb{R}_{+}^{d+1}} f(x) \Lambda(-x, y) d \mu_{\beta}(x), \quad \text { for all } y \in \mathbb{R}_{+}^{d+1} .
$$

Some basic properties of this transform are the following:

(i) For $f$ in $L_{\beta}^{1}\left(\mathbb{R}_{+}^{d+1}\right)$,

$$
\left\|\mathcal{F}_{W}(f)\right\|_{L_{\beta}^{\infty}\left(\mathbb{R}_{+}^{d+1}\right)} \leq\|f\|_{L_{\beta}^{1}\left(\mathbb{R}_{+}^{d+1}\right)}
$$

(ii) For $f$ in $\mathcal{S}_{*}\left(\mathbb{R}^{d+1}\right)$ we have

$$
\mathcal{F}_{W}\left(\triangle_{\beta} f\right)(y)=-\|y\|^{2} \mathcal{F}_{W}(f)(y), \quad \text { for all } y \in \mathbb{R}_{+}^{d+1} .
$$


(iii) For all $f$ in $L_{\beta}^{1}\left(\mathbb{R}_{+}^{d+1}\right)$, if $\mathcal{F}_{W}(f)$ belongs to $L_{\beta}^{1}\left(\mathbb{R}_{+}^{d+1}\right)$, then

$$
f(y)=C(\beta) \int_{\mathbb{R}_{+}^{d+1}} \mathcal{F}_{W}(f)(x) \Lambda(x, y) d \mu_{\beta}(x), \quad \text { a.e., }
$$

where

$$
C(\beta):=\frac{1}{\pi^{d} 4^{\beta+\frac{d}{2}}(\Gamma(\beta+1))^{2}} .
$$

(iv) For $f \in \mathcal{S}_{*}\left(\mathbb{R}^{d+1}\right)$, if we define

$$
\overline{\mathcal{F}_{W}}(f)(y)=\mathcal{F}_{W}(f)(-y),
$$

then

$$
\mathcal{F}_{W} \overline{\mathcal{F}_{W}}=\overline{\mathcal{F}_{W}} \mathcal{F}_{W}=C(\beta) I d
$$

\section{Proposition 2.1}

(i) The Weinstein transform $\mathcal{F}_{W}$ is a topological isomorphism from $\mathcal{S}_{*}\left(\mathbb{R}^{d+1}\right)$ onto itself and for all $f$ in $\mathcal{S}_{*}\left(\mathbb{R}^{d+1}\right)$,

$$
\int_{\mathbb{R}_{+}^{d+1}}|f(x)|^{2} d \mu_{\beta}(x)=C(\beta) \int_{\mathbb{R}_{+}^{d+1}}\left|\mathcal{F}_{W}(f)(\xi)\right|^{2} d \mu_{\beta}(\xi) .
$$

(ii) In particular, the renormalized Weinstein transform $f \rightarrow C(\beta)^{\frac{1}{2}} \mathcal{F}_{W}(f)$ can be uniquely extended to an isometric isomorphism from $L_{\beta}^{2}\left(\mathbb{R}_{+}^{d+1}\right)$ onto itself.

The generalized translation operator $\tau_{x}, x \in \mathbb{R}_{+}^{d+1}$, associated with the operator $\triangle_{\beta}$ is defined by

$$
\begin{aligned}
& \forall y \in \mathbb{R}_{+}^{d+1}, \\
& \tau_{x} f(y)=\frac{\Gamma(\beta+1)}{\sqrt{\pi} \Gamma\left(\beta+\frac{1}{2}\right)} \int_{0}^{\pi} f\left(x^{\prime}+y^{\prime}, \sqrt{x_{d+1}^{2}+y_{d+1}^{2}+2 x_{d+1} y_{d+1} \cos \theta}\right)(\sin \theta)^{2 \beta} d \theta,
\end{aligned}
$$

where $f \in C_{*}\left(\mathbb{R}^{d+1}\right)$.

By using the Weinstein kernel, we can also define a generalized translation. For a function $f \in \mathcal{S}_{*}\left(\mathbb{R}^{d+1}\right)$ and $y \in \mathbb{R}_{+}^{d+1}$ the generalized translation $\tau_{y} f$ is defined by the following relation:

$$
\mathcal{F}_{W}\left(\tau_{y} f\right)(x)=\Lambda(x, y) \mathcal{F}_{W}(f)(x)
$$

By using the generalized translation, we define the generalized convolution product $f * W$ $g$ of the functions $f, g \in L_{\beta}^{1}\left(\mathbb{R}_{+}^{d+1}\right)$ as follows:

$$
f *_{W} g(x)=\int_{\mathbb{R}_{+}^{d+1}} \tau_{x} f\left(-y^{\prime}, y_{d+1}\right) g(y) d \mu_{\beta}(y) .
$$

This convolution is commutative and associative and satisfies the following: 
(i) For all $f, g \in L_{\beta}^{1}\left(\mathbb{R}_{+}^{d+1}\right), f *_{W} g$ belongs to $L_{\beta}^{1}\left(\mathbb{R}_{+}^{d+1}\right)$ and

$$
\mathcal{F}_{W}\left(f *_{W} g\right)=\mathcal{F}_{W}(f) \mathcal{F}_{W}(g)
$$

(ii) Let $1 \leq p, q, r \leq \infty$ such that $\frac{1}{p}+\frac{1}{q}-\frac{1}{r}=1$. If $f \in L_{\beta}^{p}\left(\mathbb{R}_{+}^{d+1}\right)$ and $g \in L_{\beta}^{q}\left(\mathbb{R}_{+}^{d+1}\right)$, then $f *_{W} g \in L_{\beta}^{r}\left(\mathbb{R}_{+}^{d+1}\right)$ and

$$
\|f * W g\|_{L_{\beta}^{r}\left(\mathbb{R}_{+}^{d+1}\right)} \leq\|f\|_{L_{\beta}^{p}\left(\mathbb{R}_{+}^{d+1}\right)}\|g\|_{L_{\beta}^{q}\left(\mathbb{R}_{+}^{d+1}\right)}
$$

We define the tempered distribution $\mathcal{T}_{f}$ associated with $f \in L_{\beta}^{p}\left(\mathbb{R}_{+}^{d+1}\right)$ by

$$
\left\langle\mathcal{T}_{f}, \phi\right\rangle=\int_{\mathbb{R}_{+}^{d+1}} f(x) \phi(x) d \mu_{\beta}(x)
$$

for $\phi \in \mathcal{S}_{*}\left(\mathbb{R}^{d+1}\right)$ and denote by $\langle f, \phi\rangle_{\beta}$ the integral in the right hand side.

Definition 2.1 The Weinstein transform $\mathcal{F}_{W}(\tau)$ of a distribution $\tau \in \mathcal{S}_{*}^{\prime}\left(\mathbb{R}^{d+1}\right)$ is defined by

$$
\left\langle\mathcal{F}_{W}(\tau), \phi\right\rangle=\left\langle\tau, \mathcal{F}_{W}(\phi)\right\rangle
$$

for $\phi \in \mathcal{S}_{*}\left(\mathbb{R}^{d+1}\right)$.

In particular, for $f \in L_{\beta}^{p}\left(\mathbb{R}_{+}^{d+1}\right)$, it follows that for $\phi \in \mathcal{S}_{*}\left(\mathbb{R}^{d+1}\right)$,

$$
\left\langle\mathcal{F}_{W}(f), \phi\right\rangle=\left\langle\mathcal{F}_{W}\left(\mathcal{T}_{f}\right), \phi\right\rangle=\left\langle\mathcal{T}_{f}, \mathcal{F}_{W}(\phi)\right\rangle=\left\langle f, \mathcal{F}_{W}(\phi)\right\rangle_{\beta}
$$

Proposition 2.2 The Weinstein transform $\mathcal{F}_{W}$ is a topological isomorphism from $\mathcal{S}_{*}^{\prime}\left(\mathbb{R}^{d+1}\right)$ onto itself.

Definition 2.2 The generalized convolution product of a distribution $S$ in $\mathcal{S}_{*}^{\prime}\left(\mathbb{R}^{d+1}\right)$ and a function $\phi$ in $\mathcal{S}_{*}\left(\mathbb{R}^{d+1}\right)$ is the function $S *_{W} \phi$ defined by

$$
S *_{W} \phi(x)=\left\langle S_{y}, \tau_{-y} \phi(x)\right\rangle
$$

Proposition 2.3 Let $f$ be in $L_{\beta}^{p}\left(\mathbb{R}_{+}^{d+1}\right), 1 \leq p \leq \infty$, and $\phi$ in $\mathcal{S}_{*}\left(\mathbb{R}^{d+1}\right)$. Then the distribution $\mathcal{T}_{f} *_{W} \phi$ is given by the function $f *_{W} \phi$ and $\mathcal{T}_{f} *_{W} \phi$ belongs to $L_{\beta}^{p}\left(\mathbb{R}_{+}^{d+1}\right)$. Moreover, for all $\psi \in \mathcal{S}_{*}\left(\mathbb{R}^{d+1}\right)$

$$
\left\langle\mathcal{T}_{f} *_{W} \phi, \psi\right\rangle=\left\langle\check{f}, \phi *_{W} \check{\psi}\right\rangle_{\beta}
$$

where $\breve{\psi}(x)=\psi(-x)$, and

$$
\mathcal{F}_{W}\left(\mathcal{T}_{f} *_{W} \phi\right)=\mathcal{F}_{W}\left(\mathcal{T}_{f}\right) \mathcal{F}_{W}(\phi)
$$


For each $u \in \mathcal{S}_{*}^{\prime}\left(\mathbb{R}^{d+1}\right)$, we define the distribution $\triangle_{\beta} u$, by $\left\langle\triangle_{\beta} u, \psi\right\rangle=\left\langle u, \triangle_{\beta} \psi\right\rangle$. This distribution satisfies the following property:

$$
\mathcal{F}_{W}\left(\triangle_{\beta} u\right)=-\|y\|^{2} \mathcal{F}_{W}(u)
$$

In the following we denote $\mathcal{T}_{f}$ given by $(2.17)$ by $f$ for simplicity.

\section{Generalized Hardy spaces}

Definition 3.1 Let $0<p \leq 1 \leq q \leq \infty$, with $p \neq q$. A function $a \in L_{\beta}^{q}\left(\mathbb{R}_{+}^{d+1}\right)$ is a $(p, q, \beta)$ atom if $a$ satisfies the following conditions:

(i) There exist $r>0$ such that $\operatorname{supp}(a) \subset B_{+}(0, r):=\left\{x \in \mathbb{R}_{+}^{d+1}:\|x\|<r\right\}$.

(ii) $\|a\|_{L_{\beta}^{q}\left(\mathbb{R}_{+}^{d+1}\right)} \leq\left(\mu_{\beta}\left(B_{+}(0, r)\right)\right)^{\frac{1}{q}-\frac{1}{p}}$, where $r$ is given in (i).

(iii) $\int_{\mathbb{R}_{+}^{d+1}} y^{v} a(y) d \mu_{\beta}(y),|v| \leq N$, with $N=\left[(2 \beta+d+2)\left(\frac{1}{p}-1\right)\right]$ is the greatest integer not exceeding $(2 \beta+d+2)\left(\frac{1}{p}-1\right)$.

Definition 3.2 Let $0<p \leq 1$. We define the Weinstein-Hardy space $H_{\beta}^{p}\left(\mathbb{R}_{+}^{d+1}\right)$ to be the space of distributions in $\mathcal{S}_{*}^{\prime}\left(\mathbb{R}^{d+1}\right)$ such that it can be represented by

$$
f=\sum_{j=0}^{\infty} \lambda_{j} a_{j}
$$

where $\lambda_{j} \in \mathbb{C}$ such that $\sum_{j=0}^{\infty}\left|\lambda_{j}\right|^{p}<\infty$, there exist $q \in[1, \infty], q>p$ where $a_{j}$ is a $(p, q, \beta)$ atom for all $j \in \mathbb{N}$, and the series in (3.1) converges in $\mathcal{S}_{*}^{\prime}\left(\mathbb{R}^{d+1}\right)$. We define on $H_{\beta}^{p}\left(\mathbb{R}_{+}^{d+1}\right)$ the norm $\|\cdot\|_{H_{\beta}^{p}\left(\mathbb{R}_{+}^{d+1}\right)}$ by

$$
\|f\|_{H_{\beta}^{p}\left(\mathbb{R}_{+}^{d+1}\right)}=\inf \left(\sum_{j=0}^{\infty}\left|\lambda_{j}\right|^{p}\right)^{\frac{1}{p}}
$$

where the infimum is taken over all those sequences $\left(\lambda_{j}\right)_{j \in \mathbb{N}} \subset \mathbb{C}$ such that $f$ is given (3.1) for certain $(p, q, \beta)$-atoms $a_{j}, j \in \mathbb{N}$.

For the spaces $H_{\beta}^{p}\left(\mathbb{R}_{+}^{d+1}\right)$, we prove the following embedding result.

Theorem 3.1 Let $0<p \leq 1$ and $f \in H_{\beta}^{p}\left(\mathbb{R}_{+}^{d+1}\right)$, then $f \in L_{\beta}^{p}\left(\mathbb{R}_{+}^{d+1}\right)$, and

$$
\|f\|_{L_{\beta}^{p}\left(\mathbb{R}_{+}^{d+1}\right)} \leq\|f\|_{H_{\beta}^{p}\left(\mathbb{R}_{+}^{d+1}\right)} .
$$

Proof Let $a$ be a $(p, q, \beta)$-atom where $0<p \leq 1 \leq q \leq \infty$, and $p \neq q$. From the Definition 3.1, there exist $r>0$ such that

$$
a(x)=0, \quad\|x\|>r \text { and }\|a\|_{L_{\beta}^{q}\left(\mathbb{R}_{+}^{d+1}\right)} \leq\left(\mu_{\beta}\left(B_{+}(0, r)\right)\right)^{\frac{1}{q}-\frac{1}{p}} .
$$

Then from Hölder's inequality

$$
\|a\|_{L_{\beta}^{p}\left(\mathbb{R}_{+}^{d+1}\right)}=\left(\int_{\mathbb{R}_{+}^{d+1}}|a(x)|^{p} d \mu_{\beta}(x)\right)^{\frac{1}{p}} \leq\|a\|_{L_{\beta}^{q}\left(\mathbb{R}_{+}^{d+1}\right)}\left(\mu_{\beta}\left(B_{+}(0, r)\right)\right)^{\frac{1}{p}-\frac{1}{q}} \leq 1 .
$$


Now let $f \in H_{\beta}^{p}\left(\mathbb{R}_{+}^{d+1}\right)$, then $f=\sum_{j=0}^{\infty} \lambda_{j} a_{j}$, where $\lambda_{j} \in \mathbb{C}$ and $a_{j}$ is a $(p, q, \beta)$-atom, for all $j \in \mathbb{N}$, such that $\sum_{j=0}^{\infty}\left|\lambda_{j}\right|^{p}<\infty$. From the above, the series defining $f$ converges in $L_{\beta}^{p}\left(\mathbb{R}_{+}^{d+1}\right)$. Hence, $f \in L_{\beta}^{p}\left(\mathbb{R}_{+}^{d+1}\right)$ and

$$
\|f\|_{L_{\beta}^{p}\left(\mathbb{R}_{+}^{d+1}\right)} \leq \sum_{j=0}^{\infty}\left|\lambda_{j}\right|
$$

Using the fact that $\sum_{j=0}^{\infty}\left|\lambda_{j}\right| \leq\left(\sum_{j=0}^{\infty}\left|\lambda_{j}\right|^{p}\right)^{\frac{1}{p}}$, we obtain the result.

Theorem 3.2 Let $0<p \leq 1$ and $f \in H_{\beta}^{p}\left(\mathbb{R}_{+}^{d+1}\right)$, then there exists a positive constant $C$ such that

$$
\left|\mathcal{F}_{W}(f)(x)\right| \leq C\|x\|^{(2 \beta+d+2)\left(\frac{1}{p}-1\right)}\|f\|_{H_{\beta}^{p}\left(\mathbb{R}_{+}^{d+1}\right)} .
$$

Proof Let $f \in H_{\beta}^{p}\left(\mathbb{R}_{+}^{d+1}\right), 0<p \leq 1$. Then $f=\sum_{j=0}^{\infty} \lambda_{j} a_{j}$, where the series converges in $\mathcal{S}_{*}^{\prime}\left(\mathbb{R}^{d+1}\right)$ for a certain $(p, q, \beta)$-atom and $\lambda_{j} \in \mathbb{C}, j \in \mathbb{N}$. As $\mathcal{F}_{W}$ is a continuous linear mapping from $\mathcal{S}_{*}^{\prime}\left(\mathbb{R}^{d+1}\right)$ onto itself, we have

$$
\mathcal{F}_{W}(f)=\sum_{j=0}^{\infty} \lambda_{j} \mathcal{F}_{W}\left(a_{j}\right)
$$

Hence

$$
\left|\mathcal{F}_{W}(f)(x)\right| \leq \sum_{j=0}^{\infty}\left|\lambda_{j}\right|\left|\mathcal{F}_{W}\left(a_{j}\right)(x)\right| \leq \sup _{j \in \mathbb{N}}\left|\mathcal{F}_{W}\left(a_{j}\right)(x)\right| \sum_{j=0}^{\infty}\left|\lambda_{j}\right|
$$

Moreover, since $\sum_{j=0}^{\infty}\left|\lambda_{j}\right| \leq\left(\sum_{j=0}^{\infty}\left|\lambda_{j}\right|^{p}\right)^{\frac{1}{p}}$, we obtain

$$
\left|\mathcal{F}_{W}(f)(x)\right| \leq \sup _{j \in \mathbb{N}}\left|\mathcal{F}_{W}\left(a_{j}\right)(x)\right|\left(\sum_{j=0}^{\infty}\left|\lambda_{j}\right|^{p}\right)^{\frac{1}{p}}
$$

Thus it suffices to estimate $\sup _{j \in \mathbb{N}}\left|\mathcal{F}_{W}\left(a_{j}\right)(x)\right|$. Indeed, let $j \in \mathbb{N}$, we have from the properties of the $(p, q, \beta)$-atom $a_{j}$

$$
\begin{aligned}
\left|\mathcal{F}_{W}\left(a_{j}\right)(x)\right| & =\left|\int_{B_{+}(0, r)} a_{j}(y) \Lambda(-x, y) d \mu_{\beta}(y)\right| \\
& =\left|\int_{B_{+}(0, r)}\left(\Lambda(-x, y)-\sum_{j=0}^{N} \frac{\mathcal{R}_{\beta}\left(\langle-i y, x\rangle^{j}\right)}{j !}(x)\right) a_{j}(y) d \mu_{\beta}(y)\right| \\
& \leq \int_{B_{+}(0, r)}\left|\Lambda(-x, y)-\sum_{j=0}^{N} \frac{\mathcal{R}_{\beta}\left(\langle-i y, x\rangle^{j}\right)}{j !}(x)\right|\left|a_{j}(y)\right| d \mu_{\beta}(y) .
\end{aligned}
$$

On the other hand, from the definition of the transmutation operator $\mathcal{R}_{\beta}$, it is easy to obtain

$$
\left|\Lambda(-x, y)-\sum_{j=0}^{N} \frac{\mathcal{R}_{\beta}\left(\langle-i y, x\rangle^{j}\right)}{j !}(x)\right| \leq C\|x\|^{N+1}\|y\|^{N+1} .
$$


Thus, using the properties of the $(p, q, \beta)$-atom $a_{j}$ and Hölder's inequality

$$
\begin{aligned}
\left|\mathcal{F}_{W}\left(a_{j}\right)(x)\right| & \leq C\|x\|^{N+1} \int_{B_{+}(0, r)}\|y\|^{N+1}\left|a_{j}(y)\right| d \mu_{\beta}(y) \\
& \leq C\|x\|^{N+1} r^{N+1}\left\|a_{j}\right\|_{L_{\beta}^{q}\left(\mathbb{R}_{+}^{d+1}\right)}\left(\mu_{\beta}\left(B_{+}(0, r)\right)\right)^{1-\frac{1}{q}} .
\end{aligned}
$$

As

$$
\left\|a_{j}\right\|_{L_{\beta}^{q}\left(\mathbb{R}_{+}^{d+1}\right)} \leq\left(\mu_{\beta}\left(B_{+}(0, r)\right)\right)^{\frac{1}{q}-\frac{1}{p}}
$$

we obtain

$$
\forall x \in \mathbb{R}_{+}^{d+1}, \quad\left|\mathcal{F}_{W}\left(a_{j}\right)(x)\right| \leq C\|x\|^{N+1} r^{N+1+(2 \beta+d+2)\left(1-\frac{1}{p}\right)}
$$

On the other hand, we proceed as above

$$
\begin{aligned}
\left|\mathcal{F}_{W}\left(a_{j}\right)(x)\right| & \leq \int_{B_{+}(0, r)}\left|a_{j}(y)\right| d \mu_{\beta}(y) \\
& \leq\left\|a_{j}\right\|_{L_{\beta}^{q}\left(\mathbb{R}_{+}^{d+1}\right)}\left(\mu_{\beta}\left(B_{+}(0, r)\right)\right)^{1-\frac{1}{q}} \\
& \leq C r^{(2 \beta+d+2)\left(1-\frac{1}{p}\right)} .
\end{aligned}
$$

We claim that

$$
\left|\mathcal{F}_{W}\left(a_{j}\right)(x)\right| \leq C\|x\|^{-(2 \beta+d+2)\left(1-\frac{1}{p}\right)}, \quad \forall x \in \mathbb{R}_{+}^{d+1}
$$

Indeed, if $\|x\| \leq r^{-1}$ and as $(2 \beta+d+2)\left(1-\frac{1}{p}\right)+N+1>0$, we obtain

$$
\|x\|^{N+1} r^{N+1+(2 \beta+d+2)\left(1-\frac{1}{p}\right)} \leq C\|x\|^{-(2 \beta+d+2)\left(1-\frac{1}{p}\right)} .
$$

Thus from (3.3), we obtain (3.5).

On the other hand if $\|x\| \geq r^{-1}$ and as $(2 \beta+d+2)\left(1-\frac{1}{p}\right)<0$, we obtain

$$
r^{(2 \beta+d+2)\left(1-\frac{1}{p}\right)} \leq C\|x\|^{-(2 \beta+d+2)\left(1-\frac{1}{p}\right)} .
$$

Thus from (3.4), we obtain (3.5). Finally, from (3.2), we obtain

$$
\left|\mathcal{F}_{W}(f)(x)\right| \leq C\|x\|^{-(2 \beta+d+2)\left(1-\frac{1}{p}\right)}\left(\sum_{j=0}^{\infty}\left|\lambda_{j}\right|^{p}\right)^{\frac{1}{p}}
$$

which finishes the proof.

Corollary 3.3 Let $0<p \leq 1$ and $f \in H_{\beta}^{p}\left(\mathbb{R}_{+}^{d+1}\right)$, then there exist a positive constant $C$ such that

$$
\mu_{\beta}\left(\left\{x \in \mathbb{R}_{+}^{d+1}:\|x\|^{(2 \beta+d+2)\left(1-\frac{2}{p}\right)}\left|\mathcal{F}_{W}(f)(x)\right| \geq \lambda\right\}\right) \leq C \frac{\|f\|_{H_{\beta}^{p}\left(\mathbb{R}_{+}^{d+1}\right)}^{p}}{\lambda^{p}}, \quad \lambda>0 .
$$


Proof Let $0<p \leq 1$ and $f \in H_{\beta}^{p}\left(\mathbb{R}_{+}^{d+1}\right)$, and let $\lambda>0$. From Theorem 3.2, we have

$$
\begin{aligned}
& \mu_{\beta}\left(\left\{x \in \mathbb{R}_{+}^{d+1}:\|x\|^{(2 \beta+d+2)\left(1-\frac{2}{p}\right)}\left|\mathcal{F}_{W}(f)(x)\right| \geq \lambda\right\}\right) \\
& \leq \mu_{\beta}\left(B_{+}\left(0,\left(\frac{C\|f\|_{H_{\beta}^{p}\left(\mathbb{R}_{+}^{d+1}\right)}}{\lambda}\right)^{\frac{p}{2 \beta+d+2}}\right)\right) \\
& \leq C \frac{\|f\|_{H_{\beta}^{p}\left(\mathbb{R}_{+}^{d+1}\right)}^{p}}{\lambda^{p}} .
\end{aligned}
$$

Thus the proof of the corollary is complete.

\section{Hardy-type inequalities associated with the Weinstein operator}

One of the main tools in this paper is the homogeneous Littlewood-Paley decomposition of distributions associated with the Weinstein operators into dyadic blocs of frequencies.

Lemma 4.1 Let us define by $\mathcal{C}$ the ring of center 0 , of small radius 1 and great radius 2. There exist two radial functions $\psi$ and $\varphi$ the values of which are in the interval $[0,1]$ belonging to $D_{*}\left(\mathbb{R}^{d+1}\right)$ such that

$$
\begin{aligned}
& \operatorname{supp} \psi \subset B\left(0, \frac{3}{2}\right), \quad \operatorname{supp} \varphi \subset \mathcal{C}, \\
& \forall \xi \in \mathbb{R}^{d+1}, \quad \psi(\xi)+\sum_{j \geq 0} \varphi\left(2^{-j} \xi\right)=1, \\
& \forall \xi \in \mathcal{C}, \quad \sum_{j \in \mathbb{Z}} \varphi\left(2^{-j} \xi\right)=1, \\
& |n-m| \geq 2 \quad \Rightarrow \quad \operatorname{supp} \varphi\left(2^{-n} \cdot\right) \cap \operatorname{supp} \varphi\left(2^{-m} \cdot\right)=\emptyset \\
& j \geq 1 \quad \Rightarrow \quad \operatorname{supp} \psi \cap \operatorname{supp} \varphi\left(2^{-j} \cdot\right)=\emptyset .
\end{aligned}
$$

Notations We denote by

$$
\Delta_{j} f=\mathcal{F}_{W}^{-1}\left(\varphi\left(\frac{\xi}{2^{j}}\right) \mathcal{F}_{W}(f)\right), \quad S_{j} f=\sum_{n \leq j-1} \Delta_{n} f, \quad \text { for all } j \in \mathbb{Z}
$$

The distribution $\Delta_{j} f$ is called the $j$ th dyadic block of the homogeneous Littlewood-Paley decomposition of $f$ associated with the Weinstein operators.

Definition 4.1 Let us denote by $\mathcal{S}_{h, \beta, *}^{\prime}\left(\mathbb{R}^{d+1}\right)$ the space of tempered distributions such that

$$
\lim _{j \rightarrow-\infty} S_{j} u=0 \quad \text { in } \mathcal{S}_{*}^{\prime}\left(\mathbb{R}^{d+1}\right)
$$

From now on, we agree to the convention that for all non-negative sequence $\left\{a_{q}\right\}_{q \in \mathbb{Z}}$, the notation $\left(\sum_{q} a_{q}^{r}\right)^{\frac{1}{r}}$ stands for $\sup _{q} a_{q}$ in the case $r=\infty$. 
Definition 4.2 Let $s \in \mathbb{R}$ and $p, q \in[1, \infty]$. The homogeneous Weinstein-Besov space $\dot{\mathcal{B}}_{p, q}^{s, \beta}\left(\mathbb{R}_{+}^{d+1}\right)$ is the space of distributions in $\mathcal{S}_{h, \beta, *}^{\prime}\left(\mathbb{R}^{d+1}\right)$ such that

$$
\|f\|_{\mathcal{B}_{p, q}^{s, \beta}\left(\mathbb{R}_{+}^{d+1)}\right.}:=\left(\sum_{j \in \mathbb{Z}}\left(2^{s j}\left\|\Delta_{j} f\right\|_{L_{\beta}^{p}\left(\mathbb{R}_{+}^{d+1}\right)}\right)^{q}\right)^{\frac{1}{q}}<\infty .
$$

Remark 4.1 Let $p, q$ be as above. The above definition of the space $\dot{\mathcal{B}}_{p, q}^{s, \beta}\left(\mathbb{R}_{+}^{d+1}\right)$ does not depend on the choice of the couple $(\varphi, \psi)$ defining the homogeneous Weinstein-LittlewoodPaley decomposition.

Definition 4.3 For $s \in \mathbb{R}$, the operator $\mathcal{R}_{\beta}^{s}$ from $\mathcal{S}_{h, \beta, *}^{\prime}\left(\mathbb{R}^{d+1}\right)$ to $\mathcal{S}_{h, \beta, *}^{\prime}\left(\mathbb{R}^{d+1}\right)$ is defined by

$$
\mathcal{R}_{\beta}^{s}(f)=\mathcal{F}_{W}^{-1}\left(\|\cdot\|^{s} \mathcal{F}_{W} f\right)
$$

The operator $\mathcal{R}_{\beta}^{-s}$ is called a Weinstein-Riesz potential.

Definition 4.4 For $s \in \mathbb{R}$ and $1 \leq p \leq \infty$, the homogeneous Weinstein-Riesz potential space $\dot{\mathcal{H}}_{p, \beta}^{s}\left(\mathbb{R}_{+}^{d+1}\right)$ is defined as the space $\mathcal{R}_{\beta}^{-s}\left(L_{\beta}^{p}\left(\mathbb{R}_{+}^{d+1}\right)\right)$, equipped with the norm

$$
\|f\|_{\dot{\mathcal{H}}_{p, \beta}^{s}\left(\mathbb{R}_{+}^{d+1}\right)}=\left\|\mathcal{R}_{\beta}^{s}(f)\right\|_{L_{\beta}^{p}\left(\mathbb{R}_{+}^{d+1}\right)} .
$$

Theorem 4.2 Let $\frac{d+2 \beta+2}{4}<s<\frac{d+2 \beta+2}{2}$ be given. There exists a positive constant $C$ such that for all function $u \in \dot{\mathcal{H}}_{2, \beta}^{s}\left(\mathbb{R}_{+}^{d+1}\right)$ we have

$$
\int_{\mathbb{R}_{+}^{d+1}} \frac{|u(x)|^{2}}{\|x\|^{2 s}} d \mu_{\beta}(x) \leq C\|u\|_{\dot{\mathcal{H}}_{2, \beta}^{s}}^{2}\left(\mathbb{R}_{+}^{d+1}\right)
$$

For a proof this theorem we need the following lemma, which we obtain by a simple calculation.

Lemma 4.3 Let $s$ be a real number in the interval $\left(0, \beta+1+\frac{d}{2}\right)$. Then the function $x \mapsto$ $\|x\|^{-2 s}$ belongs to the Weinstein-Besov space $\dot{\mathcal{B}}_{1, \infty}^{d+2 \beta+2-2 s, \beta}\left(\mathbb{R}_{+}^{d+1}\right)$.

Proof of Theorem 4.2 Let us define

$$
I_{s, \beta}(u):=\int_{\mathbb{R}_{+}^{d+1}} \frac{|u(x)|^{2}}{\|x\|^{2 s}} d \mu_{\beta}(x)=\left\langle\|\cdot\|^{-2 s}, u^{2}\right\rangle .
$$

Using a homogeneous Littlewood-Paley decomposition and the fact that $u^{2}$ belongs to $\mathcal{S}_{h, \beta, *}^{\prime}\left(\mathbb{R}^{d+1}\right)$, we can write

$$
\begin{aligned}
I_{s, \beta}(u) & =\sum_{|n-m| \leq 2}\left\langle\Delta_{n}\left(\|\cdot\|^{-2 s}\right), \Delta_{m}\left(u^{2}\right)\right\rangle \\
& \leq C \sum_{|n-m| \leq 2}\left\langle 2^{n\left(\frac{d+2 \beta+2}{2}-2 s\right)} \Delta_{n}\left(\|\cdot\|^{-2 s}\right), 2^{-m\left(\frac{d+2 \beta+2}{2}-2 s\right)} \Delta_{m}\left(u^{2}\right)\right\rangle .
\end{aligned}
$$

Lemma 4.3 claims that $\|\cdot\|^{-2 s}$ belongs to $\dot{\mathcal{B}}_{2, \infty}^{\frac{d+2 \beta+2}{2}-2 s, \beta}\left(\mathbb{R}_{+}^{d+1}\right)$. 
Moreover, it is easy to prove that when $u$ belongs to $\dot{\mathcal{H}}_{2, \beta}^{s}\left(\mathbb{R}_{+}^{d+1}\right)$, then

$$
u^{2} \in \dot{\mathcal{B}}_{2,1}^{2 s-\frac{d+2 \beta+2}{2}, \beta}\left(\mathbb{R}_{+}^{d+1}\right) \quad \text { and } \quad\left\|u^{2}\right\|_{\dot{\mathcal{B}}_{2,1}^{2 s-\frac{d+2 \beta+2}{2}, \beta}\left(\mathbb{R}_{+}^{d+1}\right)} \leq C\|u\|_{\dot{\mathcal{H}}_{2, \beta}^{s}\left(\mathbb{R}_{+}^{d+1}\right)}^{2} \text {. }
$$

Thus

$$
I_{s, \beta}(u) \leq C\|u\|_{\dot{\mathcal{H}}_{2, \beta}^{s}\left(\mathbb{R}_{+}^{d+1}\right)^{\cdot}}^{2}
$$

For any measurable function $f$ on $\mathbb{R}_{+}^{d+1}$, we define its distribution and rearrangement functions by

$$
d_{f, \beta}(\lambda):=m_{\beta}(\{|f| \geq \lambda\}), \quad f_{\beta}^{*}(s):=\inf \left\{\lambda: d_{f, \beta}(\lambda) \leq s\right\} .
$$

For $1 \leq p<\infty$ and $1 \leq q \leq \infty$, define

$$
\|f\|_{L_{\beta}^{p, q}\left(\mathbb{R}_{+}^{d+1}\right)}= \begin{cases}\left(\int_{0}^{\infty}\left(s^{\frac{1}{p}} f_{\beta}^{*}(s)\right)^{q} \frac{d s}{s}\right)^{\frac{1}{q}} & \text { if } q<\infty \\ \sup _{s>0} s^{\frac{1}{p}} f_{\beta}^{*}(s) & \text { if } q=\infty .\end{cases}
$$

The generalized Lorentz space $L_{\beta}^{p, q}\left(\mathbb{R}_{+}^{d+1}\right)$ is defined as the set of all measurable functions $f$ such that $\|f\|_{L_{\beta}^{p, q}\left(\mathbb{R}_{+}^{d+1}\right)}<\infty$.

By a simple calculations, we prove that

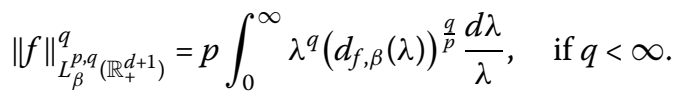

It is easy to see that $L_{\beta}^{p, p}\left(\mathbb{R}_{+}^{d+1}\right)=L_{\beta}^{p}\left(\mathbb{R}_{+}^{d+1}\right)$ and that generalized Lorentz spaces can be derived from $L_{\beta}^{p}\left(\mathbb{R}_{+}^{d+1}\right)$ spaces by the real interpolation method. In particular, when $1<p<$ $\infty$ we have $L_{\beta}^{p, q}\left(\mathbb{R}_{+}^{d+1}\right)=\left[L_{\beta}^{1}\left(\mathbb{R}_{+}^{d+1}\right), L_{\beta}^{\infty}\left(\mathbb{R}_{+}^{d+1}\right)\right]_{\theta, q}$, with $\frac{1}{p}=1-\theta$.

Theorem 4.4 Let $q \in[1, \infty]$ and let $s \in \mathbb{R}$ such that $0<s<\frac{2 \beta+d+2}{q}$, then we have

$$
\|f\|_{L_{\beta}^{p, q}\left(\mathbb{R}_{+}^{d+1}\right)} \leq C\|f\|_{\dot{\mathcal{B}}_{\infty, q}^{s-\frac{d+2 \beta}{q}, \beta}}^{1-\frac{q}{p}} \underset{\left(\mathbb{R}_{+}^{d+1}\right)}{ }\|f\|_{\dot{\mathcal{B}}_{q, q}^{s, \beta}\left(\mathbb{R}_{+}^{d+1}\right)}^{\frac{q}{d+1}},
$$

where $p=\frac{q(2 \beta+d+2)}{2 \beta+d+2-q s}$.

Proof Let $f$ be in $\mathcal{S}_{*}\left(\mathbb{R}^{d+1}\right)$; we have

$$
\|f\|_{L_{\beta}^{p, q}\left(\mathbb{R}_{+}^{d+1}\right)}^{q}=p \int_{0}^{\infty} \lambda^{q}\left(d_{f, \beta}(\lambda)\right)^{\frac{q}{p}} \frac{d \lambda}{\lambda}
$$

For $A>0$, we put $f=f_{1, A}+f_{2, A}$, with

$$
f_{1, A}=A^{d+2 \beta+2} \psi(A .) *_{W} f \quad \text { and } \quad f_{2, A}=A^{d+2 \beta+2}(I d-\psi(A .)) *_{W} f
$$

where $\psi$ is define in Lemma 4.1. 
To proceed as in [22] we prove

$$
\int_{0}^{\infty} A^{s q-d-2 \beta-3}\left\|f_{1, A}\right\|_{L_{\beta}^{\infty}\left(\mathbb{R}_{+}^{d+1}\right)}^{q} d A \leq C\|f\|_{\dot{\mathcal{B}}_{\infty, q}^{s-\frac{d+2 \beta+2}{q}, \beta}{ }_{\left(\mathbb{R}_{+}^{d+1}\right)}^{q}}^{q}
$$

and

$$
\int_{0}^{\infty} A^{s q-1}\left\|f_{2, A}\right\|_{L_{\beta}^{\infty}\left(\mathbb{R}_{+}^{d+1}\right)}^{q} d A \leq C\|f\|_{\dot{\mathcal{B}}_{q, q}^{s, \beta}\left(\mathbb{R}_{+}^{d+1}\right)}^{q}
$$

For all $\lambda>0$, we have

$$
\{|f| \geq \lambda\} \subset\left\{\left|f_{1, A}\right| \geq \frac{\lambda}{2}\right\} \cup\left\{\left|f_{2, A}\right| \geq \frac{\lambda}{2}\right\} .
$$

We take now $\lambda=\lambda(A)$ such that

$$
\left\|f_{1, A}\right\|_{L_{\beta}^{\infty}\left(\mathbb{R}_{+}^{d+1)}\right.}=\frac{\lambda}{4}
$$

Then we deduce from the choice of $\lambda$ that

$$
d_{f, \beta}(\lambda) \leq d_{f_{2, \lambda_{\lambda}, \beta}}\left(\frac{\lambda}{2}\right)
$$

By the Bienaymene-Tchebytchev inequality, we have

$$
d_{f_{2, A_{\lambda}, \beta}}\left(\frac{\lambda}{2}\right) \leq 2^{q} \lambda^{-q}\left\|f_{2, A_{\lambda}}\right\|_{L_{\beta}^{q}\left(\mathbb{R}_{+}^{d+1}\right)}^{q} .
$$

Moreover,

$$
\|f\|_{L_{\beta}^{p, q}\left(\mathbb{R}_{+}^{d+1}\right)}^{q}=p \int_{0}^{\infty} \lambda^{q}\left(d_{f, \beta}(\lambda)\right)^{\frac{q}{p}} \frac{d \lambda}{\lambda} \leq p \int_{0}^{\infty} \lambda(A)^{q-1} \lambda^{\prime}(A)\left(d_{f_{2, A_{\lambda}}, \beta}\left(\frac{\lambda}{2}\right)\right)^{\frac{q}{p}} d A .
$$

From the definitions of $\lambda$ and $f_{2, A_{\lambda}}$, we deduce

$$
\begin{aligned}
\|f\|_{L_{\beta}^{p, q}\left(\mathbb{R}_{+}^{d+1}\right)}^{q} \leq & C\left[\int_{0}^{\infty} A^{(2 \beta+d+2) q}\left\|\psi(A .) *_{W} f\right\|_{L_{\beta}^{\infty}\left(\mathbb{R}_{+}^{d+1}\right)}^{q}\left(d_{f_{2, A_{\lambda}}, \beta}\left(\frac{\lambda}{2}\right)\right)^{\frac{q}{p}} \frac{d A}{A}\right. \\
& +\int_{0}^{\infty} A^{(2 \beta+d+2)(q-1)}\left\|\psi(A .) *_{W} f\right\|_{L_{\beta}^{\infty}\left(\mathbb{R}_{+}^{d+1}\right)}^{q-1} \\
& \left.\times\left\|\Theta(A .) *_{W} f\right\|_{L_{\beta}^{\infty}\left(\mathbb{R}_{+}^{d+1}\right)}\left(d_{f_{2, A_{\lambda}, \beta}}\left(\frac{\lambda}{2}\right)\right)^{\frac{q}{p}} d A\right] \\
= & I_{1}+I_{2}
\end{aligned}
$$

where

$$
\Theta(A x)=\langle\nabla \psi(A x), x\rangle
$$


Applying the Hölder inequality, we obtain

$$
\begin{aligned}
& I_{1} \leq C\left(\int_{0}^{\infty} A^{q s-(2 \beta+d+2)}\left\|f_{1, A}\right\|_{L_{\beta}^{\infty}\left(\mathbb{R}_{+}^{d+1}\right)}^{q} \frac{d A}{A}\right)^{1-\frac{q}{p}}\left(\int_{0}^{\infty} A^{q s}\left\|f_{2, A}\right\|_{L_{\beta}^{q}\left(\mathbb{R}_{+}^{d+1}\right)}^{q} \frac{d A}{A}\right)^{\frac{q}{p}} \\
& \leq C\left(\|f\|_{\dot{\mathcal{B}}_{q, q}^{s, \beta}\left(\mathbb{R}_{+}^{d+1}\right)}^{\frac{q}{p}}\|f\|_{\dot{\mathcal{B}}_{\infty, q}^{s-\frac{d+2 \beta}{q}, \beta}\left(\mathbb{R}_{+}^{d+1}\right)}^{1-\frac{q}{p}}\right)^{q} .
\end{aligned}
$$

Proceeding in an exactly similar manner for $I_{1}$, we obtain

$$
\begin{aligned}
I_{2} \leq & C\left(\int_{0}^{\infty} A^{(2 \beta+d+2)(q-1)}\left\|\psi(A .) *_{W} f\right\|_{L_{\beta}^{\infty}\left(\mathbb{R}_{+}^{d+1}\right)}^{q-1}\left\|\Theta(A .) *_{W} f\right\|_{L_{\beta}^{\infty}\left(\mathbb{R}_{+}^{d+1}\right)} \frac{d A}{A}\right)^{\frac{q}{p}} \\
& \times\left(\int_{0}^{\infty} A^{q s}\left\|f_{2, A}\right\|_{L_{\beta}^{q}\left(\mathbb{R}_{+}^{d+1}\right)}^{q} \frac{d A}{A}\right)^{\frac{q}{p}} .
\end{aligned}
$$

By a simple calculation it is easy to obtain

$$
I_{2} \leq C\left(\|f\|_{\dot{\mathcal{B}}_{q, q}^{s, \beta}\left(\mathbb{R}_{+}^{d+1}\right)}^{\frac{q}{p}}\|f\|_{\dot{\mathcal{B}}_{\infty, q}^{s-\frac{d+2 \beta+2}{q}, \beta}\left(\mathbb{R}_{+}^{d+1}\right)}^{1-\frac{q}{p}}\right)^{q} .
$$

Combining our estimates for $I_{1}$ and $I_{2}$ we have proved that

$$
\|f\|_{L_{\beta}^{p, q}\left(\mathbb{R}_{+}^{d+1}\right)}^{q} \leq C\left(\|f\|_{\dot{\mathcal{B}}_{q, q}^{s, \beta}\left(\mathbb{R}_{+}^{d+1}\right)}^{\frac{q}{p}}\|f\|_{\mathcal{B}_{\infty, q}^{s-\frac{d+2 \beta+2}{q}, \beta}\left(\mathbb{R}_{+}^{d+1}\right)}^{1-\frac{q}{d+1}}\right)^{q},
$$

which is the desired result.

Corollary 4.5 Let s be a real number in the interval $\left(0, \frac{d+2 \beta+2}{q}\right)$ and let $q$ be a real number in $[1, \infty]$ There is a constant $C$ such that, for any function $f \in \dot{\mathcal{B}}_{q, q}^{s, \beta}\left(\mathbb{R}_{+}^{d+1}\right)$, the following inequality holds:

$$
\left(\int_{\mathbb{R}_{+}^{d+1}} \frac{|f(x)|^{q}}{\|x\|^{s q}} d \mu_{\beta}(x)\right)^{\frac{1}{q}} \leq C\|f\|_{\dot{\mathcal{B}}_{q, q}^{s, \beta}\left(\mathbb{R}_{+}^{d+1}\right)}^{\theta}\|f\|_{\dot{\mathcal{B}}_{\infty, q}^{s-\frac{d+2 \beta+2}{q}, \beta}\left(\mathbb{R}_{+}^{d+1}\right)}^{1-\theta},
$$

where $\theta=1-\frac{q s}{d+2 \beta+2}$.

For a proof of this result we need the following lemma, which we prove as in the Euclidean case.

Lemma 4.6 Let $1 \leq p_{1}, p_{2}, q_{1}, q_{2} \leq \infty$. If $\in L_{\beta}^{p_{1}, q_{1}}\left(\mathbb{R}_{+}^{d+1}\right)$ and $g \in L_{\beta}^{p_{2}, q_{2}}\left(\mathbb{R}_{+}^{d+1}\right)$, then

$$
\left.\|f g\|_{L_{\beta}^{p, q}\left(\mathbb{R}_{+}^{d+1}\right)} \leq C\|f\|_{L_{\beta}^{p_{1}, q_{1}}} \mathbb{R}_{+}^{d+1}\right)\|g\|_{L_{\beta}^{p_{2}, q_{2}}\left(\mathbb{R}_{+}^{d+1}\right)},
$$

where $\frac{1}{p}=\frac{1}{p_{1}}+\frac{1}{p_{2}}$ and $\frac{1}{q}=\frac{1}{q_{1}}+\frac{1}{q_{2}}$.

Proof of Corollary 4.5 Let as in the previous theorem $1<p<\infty$ and $s \in\left(0, \frac{d+2 \beta+2}{q}\right)$ with $\frac{1}{p}=\frac{1}{q}-\frac{s}{d+2 \beta+2}$. We take $g(x)=\frac{1}{\|x\|^{s}}$ and apply (4.7), in the specific form

$$
\left.\|f g\|_{L_{\beta}^{q, q}\left(\mathbb{R}_{+}^{d+1}\right)} \leq C\|f\|_{L_{\beta}^{p, q}\left(\mathbb{R}_{+}^{d+1}\right)}\|g\|_{L_{\beta}^{r, \infty}} \mathbb{R}_{+}^{d+1}\right)
$$


where $r=\frac{d+2 \beta+2}{s}$ and $p=\frac{q(2 \beta+d+2)}{2 \beta+d+2-q s}$. As $g \in L_{\beta}^{r, \infty}\left(\mathbb{R}_{+}^{d+1}\right)$, we have

$$
\left(\int_{\mathbb{R}_{+}^{d+1}} \frac{|f(x)|^{q}}{\|x\|^{s q}} d \mu_{\beta}(x)\right)^{\frac{1}{q}} \leq C\|f\|_{L_{\beta}^{p, q}\left(\mathbb{R}_{+}^{d+1}\right)} .
$$

Combining this with (4.3), we obtain (4.6).

Theorem 4.7 Let $0<p \leq 1$ and $N=\left[(2 \beta+d+2)\left(\frac{1}{p}-1\right)\right]$ is the greatest integer not exceeding $(2 \beta+d+2)\left(\frac{1}{p}-1\right)$. For any $f \in H_{\beta}^{p}\left(\mathbb{R}_{+}^{d+1}\right)$, we have

$$
\int_{\mathbb{R}_{+}^{d+1}} \frac{\left|\mathcal{F}_{W}(f)(x)\right|^{p}}{\|x\|^{s}} d \mu_{\beta}(x) \leq C\|f\|_{H_{\beta}^{p}\left(\mathbb{R}_{+}^{d+1}\right)}^{p}
$$

provided that

$$
(2 \beta+d+2)(2-p) \leq s<2 \beta+d+2+p(N+1) .
$$

Proof Let $f \in H_{\beta}^{p}\left(\mathbb{R}_{+}^{d+1}\right), 0<p \leq 1$. Then $f=\sum_{j=0}^{\infty} \lambda_{j} a_{j}$, where the series converges in $\mathcal{S}_{*}^{\prime}\left(\mathbb{R}^{d+1}\right)$ for a certain $(p, q, \beta)$-atom and $\lambda_{j} \in \mathbb{C}, j \in \mathbb{N}$. As $\mathcal{F}_{W}$ is a continuous linear mapping from $\mathcal{S}_{*}^{\prime}\left(\mathbb{R}^{d+1}\right)$ onto itself, we have

$$
\mathcal{F}_{W}(f)=\sum_{j=0}^{\infty} \lambda_{j} \mathcal{F}_{W}\left(a_{j}\right)
$$

Hence

$$
\int_{\mathbb{R}_{+}^{d+1}} \frac{\left|\mathcal{F}_{W}(f)(x)\right|^{p}}{\|x\|^{s}} d \mu_{\beta}(x) \leq \sum_{j=0}^{\infty}\left|\lambda_{j}\right|^{p} \int_{\mathbb{R}_{+}^{d+1}} \frac{\left|\mathcal{F}_{W}\left(a_{j}\right)(x)\right|^{p}}{\|x\|^{s}} d \mu_{\beta}(x) .
$$

Thus in order to establish the theorem, it suffice to prove

$$
\int_{\mathbb{R}_{+}^{d+1}} \frac{\left|\mathcal{F}_{W}\left(a_{j}\right)(x)\right|^{p}}{\|x\|^{s}} d \mu_{\beta}(x) \leq C .
$$

Indeed, let $R$ is a positive constant. We have

$$
\begin{aligned}
& \int_{\mathbb{R}_{+}^{d+1}} \frac{\left|\mathcal{F}_{W}\left(a_{j}\right)(x)\right|^{p}}{\|x\|^{s}} d \mu_{\beta}(x) \\
& \quad=\int_{B_{+}(0, R)} \frac{\left|\mathcal{F}_{W}\left(a_{j}\right)(x)\right|^{p}}{\|x\|^{s}} d \mu_{\beta}(x)+\int_{\mathbb{R}_{+}^{d+1} \backslash B_{+}(0, R)} \frac{\left|\mathcal{F}_{W}\left(a_{j}\right)(x)\right|^{p}}{\|x\|^{s}} d \mu_{\beta}(x) \\
& \quad:=I_{1}+I_{2} .
\end{aligned}
$$

From (3.3) and (4.9), we have

$$
\begin{aligned}
I_{1} & \leq C r^{p(2 \beta+d+3+N)-(2 \beta+d+2)} \int_{B_{+}(0, R)} \frac{\|x\|^{p(N+1)}}{\|x\|^{s}} d \mu_{\beta}(x) \\
& \leq C r^{p(2 \beta+d+3+N)-(2 \beta+d+2)} R^{2 \beta+d+2+p(N+1)-s} .
\end{aligned}
$$


Now we will estimate $I_{2}$. Indeed, we apply Hölder's inequality and Plancherel's formula, and we use (4.9), and we obtain

$$
\begin{aligned}
I_{2} & \leq\left(\int_{\mathbb{R}_{+}^{d+1}}\left|\mathcal{F}_{W}\left(a_{j}\right)(x)\right|^{2} d \mu_{\beta}(x)\right)^{\frac{p}{2}}\left(\int_{\mathbb{R}_{+}^{d+1} \backslash B_{+}(0, R)}\|x\|^{\frac{2 s}{p-2}} d \mu_{\beta}(x)\right)^{\frac{2-p}{2}} \\
& \leq C\left\|a_{j}\right\|_{L_{\beta}^{2}\left(\mathbb{R}_{+}^{d+1}\right)}^{p} R^{\frac{(2 \beta+d+2)(2-p)}{2}-s} .
\end{aligned}
$$

Moreover, from the properties of $a_{j}$, it is easy to see that

$$
\left\|a_{j}\right\|_{L_{\beta}^{2}\left(\mathbb{R}_{+}^{d+1}\right)}^{p} \leq C r^{-\frac{(2 \beta+d+2)(2-p)}{2}}
$$

Thus

$$
I_{2} \leq C r^{-\frac{(2 \beta+d+2)(2-p)}{2}} R^{\frac{(2 \beta+d+2)(2-p)}{2}-s} \text {. }
$$

To conclude we distinguish two cases.

First case: If $s=(2 \beta+d+2)(2-p)$. We put $R=\frac{1}{r}$, and we obtain $I_{1} \leq C$ and $I_{2} \leq C$.

Second case: If $(2 \beta+d+2)(2-p)<s<2 \beta+d+2+p(N+1)$. We shall discuss the cases $0<r<1$ and $r \geq 1$.

Indeed, if $0<r<1$, it is easy to see from the definition of $N$ and the condition

$$
(2 \beta+d+2)(2-p)<s<2 \beta+d+2+p(N+1)
$$

that there exists $R>0$ such that

$$
r^{p(2 \beta+d+3+N)-(2 \beta+d+2)} R^{2 \beta+d+2+p(N+1)-s} \leq C \quad \text { and } \quad r^{-\frac{(2 \beta+d+2)(2-p)}{2}} R^{\frac{(2 \beta+d+2)(2-p)}{2}-s} \leq C .
$$

Hence we obtain $I_{1} \leq C$ and $I_{2} \leq C$.

If $r \geq 1$. We put $R=r^{\frac{2 \beta+d+2-p(N+3+2 \beta+d)}{2 \beta+d+2+p(N+1)-s}}$, and it is easy to see that $I_{1} \leq C$ and $I_{2} \leq C$. Thus the proof of the theorem is finished.

\section{Competing interests}

The author declares that there is no conflict of interests regarding the publication of this paper.

\section{Acknowledgements}

The author gratefully acknowledges the Deanship of Scientific Research at the University of Taibah University on material and moral support. The author thanks Professor Khalifa Trimèche and the referees for their suggestions and comments.

Received: 5 June 2015 Accepted: 5 August 2015 Published online: 04 September 2015

\section{References}

1. Coifman, RR: A real variable characterization of $H^{p}$. Stud. Math. 51, 269-274 (1974)

2. Fefferman, C, Stein, EM: HP Spaces of several variables. Acta Math. 129, 137-193 (1972)

3. Stein, EM, Weiss, GL: On the theory of harmonic functions of several variables I (the theory of $H^{p}$ spaces). Acta Math. 103, 25-62 (1960)

4. Coifman, RR, Weiss, GL: Extensions of Hardy spaces and their use in analysis. Bull. Am. Math. Soc. 83, 569-615 (1977)

5. Uchiyama, A: A maximal function characterization of $H^{P}$ on the space of homogeneous type. Trans. Am. Math. Soc. 262(2), 579-592 (1980)

6. Stein, EM: Harmonic Analysis: Real-Variable Methods, Orthogonality, and Oscillatory Integrals. Princeton Math. Ser., vol. 43. Princeton University Press, Princeton (1993)

7. Anker, J-P, Ben Salem, N, Dziubański, J, Hamda, N: The Hardy space $H^{1}$ in the rational Dunkl setting. Constr. Approx. (2014). doi:10.1007/s00365-014-9254-2 
8. Kanjin, Y: Hardy's inequalities for Hermite and Laguerre expansions. Bull. Lond. Math. Soc. 29(3), 331-337 (1997)

9. Persson, LE, Ragusa, MA, Samko, N, Wall, P: Commutators of Hardy operators in vanishing Morrey spaces. In: 9th International Conference on Mathematical Problems in Engineering, Aerospace and Sciences (ICNPAA 2012). AIP Conference Proceedings, vol. 1439, pp. 859-866 (2012). doi:10.1063/1.4765588

10. Radha, R: Hardy-type inequalities. Taiwan. J. Math. 4(3), 447-456 (2000)

11. Colzani, L, Travaglini, G: Hardy-Lorentz spaces and expansions in eigenfunctions of the Laplace-Beltrami operator on compact manifolds. Colloq. Math. 58, 305-316 (1990)

12. Radha, R, Thangavelu, S: Hardy's inequalities for Hermite and Laguerre expansions. Proc. Am. Math. Soc. 132(12), 3525-3536 (2004)

13. Ben Nahia, Z, Ben Salem, N: Spherical harmonics and applications associated with the Weinstein operator. In: Potential Theory - ICPT 94, vol. 94, pp. 233-242 (1996)

14. Brelot, M: Equation de Weinstein et potentiels de Marcel Riesz. In: Séminaire de Théorie du Potentiel, No. 3 (Paris, 1976/1977). Lecture Notes in Math., vol. 681, pp. 18-38 (1978)

15. Ben Nahia, Z, Ben Salem, N: On a mean value property associated with the Weinstein operator. In: Potential Theory ICPT 94, vol. 94, pp. 243-254 (1996)

16. Othmani, Y, Trimèche, K: Real Paley-Wiener theorems associated with the Weinstein operator. Mediterr. J. Math. 3(1), 105-118 (2006)

17. Chettaoui, C, Trimèche, K: Bockner-Hecke theorems for the Weinstein transform and applications. Fract. Calc. Appl. Anal. 13(3), 261-280 (2010)

18. Mejjaoli, H, Salhi, M: Uncertainty principles associated with the Weinstein transform. Czechoslov. Math. J. 61(4), 941-974 (2011)

19. Mejjaoli, H, Salem, AOA: Weinstein Gabor transform and applications. Adv. Pure Appl. Math. 2(3), $203-210$ (2012)

20. Ben Salem, N, Nasr, AR: Heisenberg-type inequalities for the Weinstein operator. Integral Transforms Spec. Funct. (2015). doi:10.1080/10652469.2015.1038531

21. Mejjaoli, H: Heat equations associated with Weinstein operator and applications. J. Funct. Spaces Appl. 2013, Article ID $723976(2013)$

22. Triebel, H: Interpolation Theory, Function Spaces Differential Operators. North-Holland, Amsterdam (1978)

\section{Submit your manuscript to a SpringerOpen ${ }^{\circ}$ journal and benefit from:}

- Convenient online submission

Rigorous peer review

- Immediate publication on acceptance

Open access: articles freely available online

- High visibility within the field

- Retaining the copyright to your article 